\title{
Can We Kill Two Birds With One Stone? Achieving Function and Aesthetics by Extracorporeal Septoplasty
}

\author{
Yong Ju Jang (10) \\ Department of Otorhinolaryngology-Head and Neck Surgery, Asan Medical Center, University of Ulsan College of Medicine, Seoul, Korea
}

The vast majority of patients with deviated nose experience impaired nasal breathing function. From the aesthetic standpoint, deviated nose in its various forms is one of the most difficult deformities to correct through rhinoplasty. In patients with deviated nose, proper septal correction is essential for successful treatment, as the nasal septum plays a central role in the normal function and shape of the nose. The conventional way to correct deviated nose is the combination of in situ septal correction (ISSC) and additional tip procedures, with or without dorsal augmentation. Many ISSC techniques have been used to correct severe deviation of the nasal septum. However, to correct an extremely deviated septum, extracorporeal septoplasty (ECS) has proven to be more effective. The ECS technique, in its original description, is characterized by extensive removal of the whole septum, including cartilage and bone, and reimplantation of the extracorporeally reshaped septum back into the mucosal pocket [1].

Owing to its aggressive nature and increased risk of keystone breakdown, ECS has been modified over time. One of the issues that has been addressed is stabilization of the reinserted septum. Rezaeian et al. [2] recently introduced the technique of criss-cross or transcutaneous transosseous cerclage suture to facilitate stabilization and reduce the complication rate after ECS. Jang and Kwon [3] proposed a modified ECS method, in which a small piece of cartilage is preserved at the keystone area as an anchoring part for the neo-septum; this technique has been proven to be effective for the correction of different types of deviations, entails a lower risk of keystone breakdown, and is easier to perform.

In the current issue of Clinical and Experimental Otorhinolaryngology, Mun et al. [4] presented favorable effects of modified ECS in terms of tip aesthetics and functional outcomes. The favorable effect of ECS on functional improvement reported by the authors [4] is not a completely new finding, as Lee and Jang
[5] previously reported that ECS brought about better functional outcomes than ISSC. Although Mun et al. [4] did not provide a direct comparison of functional outcomes between ECS and ISSC, their results corroborate the superior functional performance of the modified ECS technique [5]. The favorable effect of ECS on nasal aesthetics beyond the correction of deviation is an unarguably anticipated result if the surgeon understands the role of the septal cartilage in determining the tip height and dorsal height.

Individuals with prominent septal cartilage display a well-projected nasal tip and cartilaginous dorsum. ECS makes it possible to alter the dorsal height and tip dimension if the surgeon intentionally designs the neo-septum with an increased height. Thus, the aesthetic effect of modified ECS on the nasal tip and dorsum may be compatible with combining ISSC with additional tip and dorsal procedures. The meaningful improvements in shape and function attainable by a single ECS procedure with no need for additional rhinoplasty procedures make it an attractive option to offer patients who have a severely deviated nose. Indeed, ECS can achieve function and aesthetics simultaneously with one procedure.

\section{CONFLICT OF INTEREST}

No potential conflict of interest relevant to this article was reported.

\section{ORCID}

Yong Ju Jang https://orcid.org/0000-0001-7631-0388

Copyright (C) 2021 by Korean Society of Otorhinolaryngology-Head and Neck Surgery.

This is an open-access article distributed under the terms of the Creative Commons Attribution Non-Commercial License (https://creativecommons.org/licenses/by-nc/4.0)

which permits unrestricted non-commercial use, distribution, and reproduction in any medium, provided the original work is properly cited. 


\section{REFERENCES}

1. Gubisch W.Twenty-five years experience with extracorporeal septoplasty. Facial Plast Surg. 2006 Nov;22(4):230-9.

2. Rezaeian F, Gubisch W, Janku D, Haack S. New suturing techniques to reconstruct the keystone area in extracorporeal septoplasty. Plast Reconstr Surg. 2016 Aug;138(2):374-82.

3. Jang YJ, Kwon M. Modified extracorporeal septoplasty technique in rhinoplasty for severely deviated noses. Ann Otol Rhinol Laryngol. 2010 May;119(5):331-5.

4. Mun SJ, Choi YS, Kim JH, Shim WS, Jung HJ. Evaluation of the es- thetic and functional outcomes of extracorporeal septoplasty for rhinoplasty in Asian patients. Clin Exp Otorhinolaryngol. 2021 Feb; 14(1):100-7.

5. Lee SB, Jang YJ. Treatment outcomes of extracorporeal septoplasty compared with in situ septal correction in rhinoplasty. JAMA Facial Plast Surg. 2014 Sep-Oct;16(5):328-34.

Received December 16, 2020 Accepted December 17, 2020 